\title{
Erratum to: Charcot-Marie-Tooth disease type 2C and scapuloperoneal muscular atrophy overlap syndrome in a patient with the R232C TRPV4 mutation
}

\author{
Georgios Koutsis ${ }^{1}$ - David Lynch ${ }^{2} \cdot$ Andreea Manole $^{2} \cdot$ Georgia Karadima $^{1}$ •
}

Mary M. Reilly ${ }^{3} \cdot$ Henry Houlden ${ }^{2} \cdot$ Marios Panas $^{1}$

Published online: 11 July 2015

(C) Springer-Verlag Berlin Heidelberg 2015

\section{Erratum to: J Neurol \\ DOI 10.1007/s00415-015-7800-x}

In the original article, one of the co-author's (Andreea

Manole) family name has been published incorrectly.

The correct family name should be Manole.

The online version of the original article can be found under doi:10.1007/s00415-015-7800-x.

Georgios Koutsis

gkoutsi2@otenet.gr; gkoutsis@med.uoa.gr

1 Neurogenetics Unit, 1st Department of Neurology, Eginition

Hospital, University of Athens Medical School, 74 Vas.

Sophias Ave., 11528 Athens, Greece

2 Department of Molecular Neuroscience, UCL Institute of Neurology, London, UK

3 MRC Centre for Neuromuscular Diseases, UCL Institute of Neurology and the National Hospital for Neurology and Neurosurgery, London, UK 